\title{
Early Intervention in Smoldering Myeloma
}

\author{
An Expert Interview with Shaji K Kumar
}

Mayo Clinic, Rochester, MN, USA

DOI: https://doi.org/10.17925/OHR.2019.15.1.14

\section{Shaji K Kumar}

Shaji K Kumar, MD, is a consultant in the Division of Hematology at Mayo Clinic, and professor of medicine in the College of Medicine, Mayo Clinic, Rochester, MN, USA. A significant component of Dr Kumar's research efforts is directed toward studying novel treatment (a)tiot approaches in myeloma through well-designed clinical trials. He is principal investigator on multiple phase I, II, and III trials for plasma cell malignancies, which include a combination of Mayo investigator-initiated trials, industry-sponsored trials, and cooperative group trials. From a translational research standpoint, his laboratory has focused on the development of new agents for plasma cell malignancies, with a particular focus on the tumor microenvironment. Dr Kumar receives ongoing support for research from the National Institutes of Health and the National Cancer Institute. He is a member of several professional societies, including the American Society of Hematology, American Society of Clinical Oncology, American Association for Clinical Research, American Society for Blood and Marrow Transplantation, American Medical Association, Association of Physicians of India, and the European Hematology Association. His work has appeared in peer-reviewed journals such as Lancet Oncology, Journal of Clinical Oncology, Blood Cancer Journal, Leukemia, American Journal of Hematology, Mayo Clinic Proceedings, Blood, and European Journal of Hematology. He received his medical degree from the All India Institute of Medical Sciences, New Delhi, India. He completed an internship there, followed by a clinical residency in internal medicine and a senior residency in medical oncology. Dr Kumar subsequently served a residency in internal medicine and a fellowship in hematology/oncology at the Mayo Graduate School of Medicine. He then served as a research associate and Mayo Foundation Scholar in multiple myeloma at the Dana-Farber Cancer Institute, Harvard Cancer Center in Boston, MS, USA.

\section{Keywords}

Smoldering multiple myeloma, pembrolizumab, isatuximab, anti-CD 3

Disclosures: Shaji K Kumar has nothing to disclose in relation to this article.

Review Process: This is an expert interview and, as such, has not undergone the journal's standard peer review process.

Acknowledgments: Medical writing assistance was provided by Katrina Mountfort of Touch Medical Media, and supported by Touch Medical Media.

Authorship: The named author meets the Internationa Committee of Medical Journal Editors (ICMJE) criteria for authorship of this manuscript, takes responsibility for the integrity of the work as a whole, and has given final approval for the version to be published.

Received: February 7, 2019

Published Online: March 29, 2019

Citation: Oncology \& Hematology Review. 2019;15(1):14-5

Corresponding Author: Shaji K Kumar, Division of Hematology, Mayo Clinic, 200 First Street SW, Rochester, MN 55905, USA. E: kumar.shaji@mayo.edu

Support: No funding was received in

the publication of this article.
S moldering myeloma is an asymptomatic clonal plasma cell disorder that is characterized by the presence of $\geq 3 \mathrm{~g} / \mathrm{dl}$ serum M-protein and/or 10-59\% bone marrow plasma cell infiltration, and is a precursor stage to multiple myeloma. ${ }^{1}$ Historically, patients with smoldering myeloma have not received treatment until symptomatic disease has developed because many of them have a low risk of progression to myeloma within 5 years. However, a high-risk smoldering myeloma group can be identified, and may benefit from earlier intervention. ${ }^{2.3} \mathrm{In}$ an expert interview, Dr Shaj $\mathrm{K}$ Kumar discusses the risk factors for progression of smoldering myeloma to multiple myeloma and preventative strategies to avoid this progression.

\section{Q. What percentage of patients with smoldering myeloma go on to develop multiple myeloma, and what are the known risk factors for progression?}

Nearly half of patients with smoldering multiple myeloma will progress to active myeloma in the 5 years after the diagnosis. Another $15-20 \%$ will have disease progression during the subsequent 5 years. ${ }^{1}$ Of the remaining patients there is a steady risk of progression of about $1 \%$ per year which is similar to what we see in monoclonal gammopathy of undetermined significance. ${ }^{1.2}$ Over the years, multiple factors have been identified that can predict the risk of progression in patients with smoldering multiple myeloma. These include the type of monoclonal protein, the size of the monoclonal protein, serum free light chain ratio, the presence of immunoparesis, abnormalities on imaging studies, presence of circulating plasma cells, and specific genetic abnormalities, among others. 'Recent studies have continued to develop clinically usable risk stratification systems such as the International Myeloma Working Group classification system for smoldering myeloma. ${ }^{4}$

\section{Q. What is the current standard of care for smoldering myeloma?}

The current standard of care for smoldering multiple myeloma is careful and close observation. When patients are first diagnosed with smoldering myeloma, it is hard to distinguish between disease that is rapidly evolving versus true smoldering multiple myeloma. We recommend repeat 
testing approximately 3 months later to obtain a good sense of the trend. If there is stability in the disease, then these patients can be followed every 3 months. Once patients have been followed for 5 years or more with no significant change, the frequency of observation can be reduced. Beyond 10 years, patients have a much lower risk of progression and can be followed in a similar way to our approach for monoclonal gammopathy of undetermined significance.

\section{Q. What have been the findings to date of studies investigating pembrolizumab as a prevention strategy to avoid progression to multiple myeloma among patients with smoldering myeloma?}

Pembrolizumab has been studied in combination with immunomodulatory drugs like lenalidomide and pomalidomide in patients with multiple myeloma. In two different phase III trials, this drug did not demonstrate any efficacy and these trials were closed..$^{5}$ At this time, it is being studied in combination with other drugs, but there is no clear activity so far. One can hypothesize that early intervention with a checkpoint inhibitor might enhance the immune response in a beneficial manner and prevent disease progression. However, we do not have any proof at this time.

\section{Q. What have been the findings to date of the study investigating the anti-CD38 antibody isatuximab in smoldering myeloma?}

These studies are still in their infancy. Daratumumab is another anti-CD38 antibody that is currently being investigated in phase III trials in patients with smoldering multiple myeloma, both alone and in combination with lenalidomide (ClinicalTrials.gov Identifer: NCT03301220). ${ }^{6}$

\section{Q. What other investigational strategies appear promising?}

Lenalidomide and dexamethasone was studied in patients with high-risk smoldering multiple myeloma by a Spanish group.? They demonstrated an improved overall survival in patients with high-risk smoldering multiple myeloma with early intervention. A subsequent trial has compared lenalidomide alone with observation, and the results of the trial should be available in the near future. There are several phase III trials that are ongoing or being planned that will examine the role of monoclonal antibodies and oral proteasome inhibitors in patients with smoldering multiple myeloma. Other studies are looking at better understanding the biology of progression and identification of disease targets that might be amenable to therapeutic intervention.
1. Kyle RA, Remstein ED, Therneau TM, et al. Clinical course and prognosis of smoldering (asymptomatic) multiple myeloma. N Engl J Med. 2007;356:2582-90.

2. Perez-Persona $E$, Vidriales $M B$, Mateo $G$, et al. New criteria to identify risk of progression in monoclonal gammopathy of uncertain significance and smoldering multiple myeloma based on multiparameter flow cytometry analysis of bone marrow plasma cells. Blood. 2007;110:2586-92.

3. Mateos MV, Gonzalez-Calle V. Smoldering multiple myeloma: who and when to treat. Clin Lymphoma Myeloma Leuk. 2017;17:716-22.
4. Rajkumar SV. Updated diagnostic criteria and staging system for multiple myeloma. Am Soc Clin Oncol Educ Book. 2016;35:e418-23.

5. Jelinek T, Paiva B, Hajek R. Update on PD-1/PD-L1 inhibitors in multiple myeloma. Front Immunol. 2018;9:2431.

6. ClinicalTrials.gov. A Study of Subcutaneous Daratumumab versus Active Monitoring in Participants With High-Risk Smoldering Multiple Myeloma. ClinicalTrials.gov Identifier NCT03301220. Available at: https://clinicaltrials.gov/ct2/ show/NCT03301220 (accessed February 11, 2019).
7. Mateos MV, Hernandez MT, Giraldo P, et al. Long term follow-up on the tretament of high risk smoldering myeloma with lenalidomide plus low dose dex (Rd) (phase III Spanish trial): Persistent benefit in overall survival. Blood. 2014:124:3465.

8. Kumar S. Emerging options in multiple myeloma: targeted immune, and epigenetic therapies. Hematology Am Soc Hematol Educ Program. 2017;2017:518-24.

9. Morandi F, Horenstein AL, Costa F, et al. CD38: A target for immunotherapeutic approaches in multiple myeloma. Front Immunol. 2018;9:2722. 